\title{
Trichoscopy of Tinea Capitis: A Systematic Review
}

\author{
Anna Waśkiel-Burnat (D) - Adriana Rakowska (D) - Mariusz Sikora (D) · \\ Piotr Ciechanowicz (D) - Małgorzata Olszewska (D) - Lidia Rudnicka (D)
}

Received: October 1, 2019 / Published online: January 6, 2020

(C) The Author(s) 2020

\begin{abstract}
Introduction: An increased incidence of tinea capitis has been observed over the last few decades. Trichoscopy is a non-invasive, in-office method helpful in establishing the correct diagnosis in patients with hair loss and inflammatory hair disorders. The objective was to review and analyze current data on the trichoscopy of tinea capitis.

Methods: A systematic review of the literature was conducted using the PubMed, EBSCO and Scopus databases. The search terms included 'tinea capitis' combined with 'trichoscopy',
\end{abstract}

Enhanced Digital Features To view enhanced digital features for this article go to https://doi.org/10.6084/ m9.figshare. 11397753 .

A. Waśkiel-Burnat · A. Rakowska (凹) · M. Sikora · P. Ciechanowicz · M. Olszewska · L. Rudnicka Department of Dermatology, Medical University of Warsaw, Warsaw, Poland

e-mail: adriana.rakowska@gmail.com 'dermatoscopy', 'dermoscopy', 'videodermatoscopy' or 'videodermoscopy'.

Results: Of 326 articles, 37 were considered eligible for the quantitative analysis. The most characteristic (with a high predictive value) trichoscopic findings of tinea capitis included comma hairs $(51 \%)$, corkscrew hairs $(32 \%)$, Morse code-like hairs (22\%), zigzag hairs (21\%), bent hairs (27\%), block hairs (10\%) and i-hairs (10\%). Other common, but not characteristic, trichoscopic features were broken hairs (57\%), black dots (34\%), perifollicular scaling (59\%) and diffuse scaling (89\%). Morse code-like hairs, zigzag hairs, bent hairs and diffuse scaling were only observed in Microsporum tinea capitis (8/ $29,28 \% ; 6 / 29,21 \% ; 4 / 29,14 \%$ and $4 / 29,14 \%$, respectively). In Trichophyton tinea capitis, corkscrew hairs were more commonly detected compared to Microsporum tinea capitis (21/38, $55 \%$ vs $3 / 29,10 \%)$.

Conclusion: The presence of characteristic trichoscopic features of tinea capitis is sufficient to establish the initial diagnosis and introduce treatment before culture results are available. Trichoscopy may be useful in distinguishing between Microsporum and Trichophyton tinea capitis.

Keywords: Alopecia; Dermatoscopy; Dermoscopy; Hair disorders; Hair loss; Tinea; Tinea capitis; Trichoscopy; Scalp; Fungal infection 


\section{Key Summary Points}

During the last few decades, an increased prevalence of tinea capitis with a remarkable change in the pattern of the causative dermatophytes among different countries has been observed.

Although mycological examination is considered to be the gold standard diagnostic method in tinea capitis, it is highly valuable to find a method useful in making a correct diagnosis before culture results are available.

The aim of the study was to establish the role of trichoscopy in diagnosing tinea capitis.

Trichoscopy is helpful in diagnosis of tinea capitis, differentiation between

Microsporum and Trichophyton infection, and monitoring of treatment efficacy.

The presence of characteristic trichoscopic features of tinea capitis is sufficient to establish the initial diagnosis and introduce treatment before culture results are available.

\section{INTRODUCTION}

Tinea capitis is a cutaneous fungal infection or dermatophytosis of the scalp [1]. During the last few decades, an increased prevalence of the disease with a remarkable change in the pattern of the causative dermatophytes among different countries has been observed, probably due to immigration, emigration, traveling and changes in the level of surveillance [2]. Nevertheless, tinea capitis is mainly caused by Microsporum and Trichophyton species [3], its etiological agents differ according to the geographical distribution $[2,4]$. The disease is most commonly observed in children between 3 and 7 years of age [5]. Adults (especially elderly individuals) may be occasionally affected [4]. Clinically, tinea capitis is characterized by the presence of hair loss areas with coexistent scaling, inflammation or pustules [4]. Mycological examination is considered to be the gold standard diagnostic method in tinea capitis [6]. However, trichoscopy may be useful in making a correct diagnosis before culture results are available [6]. It has been reported, that trichoscopy, an easy to perform, non-invasive method $[7,8]$, is characterized by a higher sensitivity compared with direct examination (94\% vs $49.1 \%)$ in diagnosis of tinea capitis [9]. Moreover, a high specificity of trichoscopy in tinea capitis has been described (83\%) [9]. Slowinska et al. [10] first described the trichoscopic features of tinea capitis in 2008. Since then, numerous studies have been published that addressed the role of trichoscopy in the diagnosis and therapeutic monitoring of tinea capitis.

\section{METHODS}

A review of the literature regarding trichoscopic findings of tinea capitis was performed by searching the PubMed, Scopus and EBSCO databases. The terms used for the search were: 'tinea capitis' combined with 'trichoscopy', 'dermatoscopy', 'dermoscopy', 'videodermatoscopy' or 'videodermoscopy'. The references of all relevant articles were also searched for further publications. Original studies, case series and case reports considering the frequency of various trichoscopic findings in tinea capitis and published in English were eligible for the analysis. Studies with no primary epidemiologic data reporting the numbers of patients with tinea capitis or the frequency of various trichoscopic findings, animal studies and reviews were excluded from this analysis. If data were duplicated in more than one study, the most complete study was included in the analysis.

Based on the results of original studies, an analysis of the frequency of the most common trichoscopic findings of tinea capitis was performed. The sensitivity, specificity, and positive and negative predictive values of the most characteristic trichoscopic findings for tinea 
capitis were calculated and presented as percentages.

Based on the results of original studies, case series and case reports, trichoscopic differences between Microsporum and Trichophyton tinea capitis were assessed.

The role of trichoscopy in the monitoring of treatment efficacy in tinea capitis was described.

Statistical analysis of the data was conducted using Statistica software, version 12.0. The differences in the incidence rate of various trichoscopic features between Microsporum and Trichophyton tinea capitis were examined with a chi-squared test. The results were considered statistically significant if they had $p$ values lower than 0.05 .

This article is based on previously conducted studies and does not contain any studies with human participants or animals performed by any of the authors.

\section{RESULTS}

Of 326 articles retrieved, 37 studies (consisting of 536 patients with tinea capitis) were found eligible for quantitative analysis: 16 original studies, 7 case series and 14 case reports. A PRISMA flow diagram is presented in Fig. 1.

The frequency, sensitivity, specificity, and positive and negative predictive values of the most characteristic trichoscopic findings for tinea capitis are presented in Table 1.

\section{The Most Characteristic Trichoscopic Findings of Tinea Capitis}

\section{Comma Hairs}

Comma hairs (Fig. 2) are short, C-shaped hairs, that are homogeneous in pigmentation and thickness [11]. They were first described by Slowinska et al. in 2008 [10]. Comma hairs are formed due to subsequent cracking and bending of a hair shaft filled with hyphae [10]. The frequency of comma hairs varied between 13\% and $100 \%$ (mean value: $51 \%$ ) of patients with tinea capitis $[6,9,12-24]$. They were also occasionally detected in patients with alopecia areata and trichotillomania [16].

\section{Corkscrew Hairs}

Corkscrew hairs (Fig. 3) are multiple twisted and coiled hairs with corkscrew-like structure [11]. They were first described by Hughes et al. [23] in 2011 as a specific form of comma hairs in blackskinned patients (with African hair types) or a specific trichoscopic finding of tinea capitis caused by Trichophyton soudanense. However, corkscrew hairs were also described in white-skinned patients [25-27] with straight hair [28]. They were observed in endothrix tinea capitis caused by Trichophyton tonsurans $[22,26-31]$ and Trichophyton violaceum $[25,32,33]$. Moreover, corkscrew hairs were detected in ectothrix-type fungal infection caused by Trichophyton verrucosum [12], Microsporum canis and Microsporum audouinii [22]. The incidence of corkscrew hairs varied between $14 \%$ and $100 \%$ (mean value: $32 \%$ ) of patients with tinea capitis $[6,9,12-24]$. Corkscrew hairs were described as a specific trichoscopic feature of tinea capitis. However, they may also be observed in ectodermal dysplasias [34, 35].

\section{Morse Code-like Hairs}

Morse code-like hairs (Fig. 3), also known as bar code-like hairs, represent hairs with multiple thin white bands across the hair shaft [11]. The term was introduced in 2011 by Rudnicka et al. [34]. Morse code-like hairs are formed due to the accumulation of spores around the hair shaft that cause a transverse perforation of the hair shaft. They were only described in patients with ectothrix-type fungal infection with an incidence rate between $12 \%$ and $56 \%$ (mean value: $22 \%)[9,22,36-40]$.

\section{Zigzag Hairs}

Zigzag hairs, first described by Rudnicka et al. [34], are bent hairs with multiple sharp angles. Their formation results from incomplete, transverse fractures along the hair shaft [31]. Zigzag hairs were only described in patients with ectothrix-type fungal infection with an incidence rate between $5 \%$ and $49 \%$ (mean value: $21 \%)[6,9,13,15,18-21,24]$. They were also reported in patients with alopecia areata [41]. 


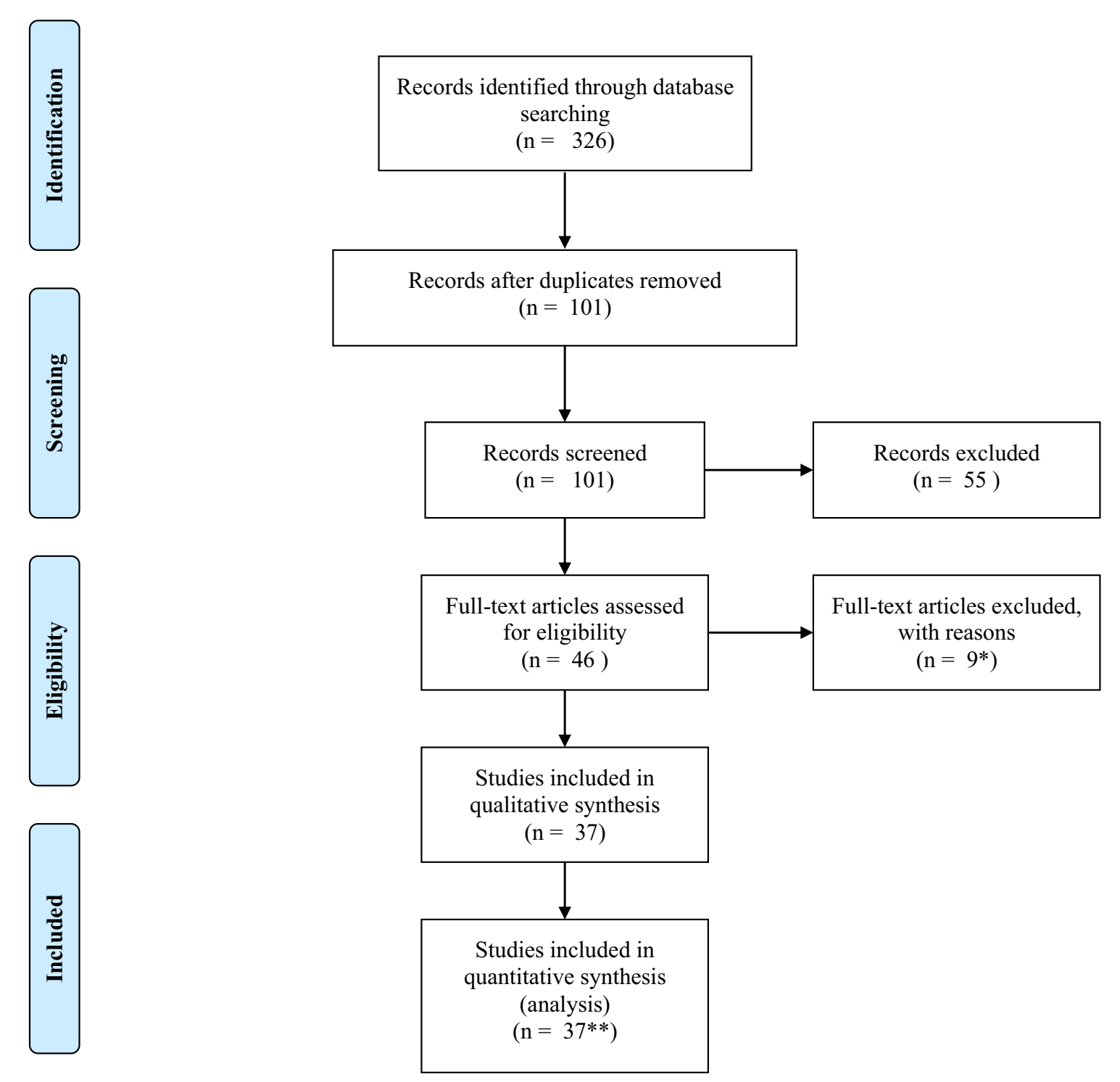

\footnotetext{
* Excluded articles: 3 articles did not consider the frequency of various trichoscopic findings, 2 articles were reviews, 2 articles were duplications, 1 study did not contain the frequency of various trichoscopic findings, 1 case series with patients with coexisted different hair diseases ** 16 original studies, 14 case reports and 7 case series
}

Fig. 1 PRISMA flow diagram

\section{Bent Hairs}

Bent hairs are characterized by bending of the hair shaft with homogeneous thickness and pigmentation [42]. In contrast to comma hairs, no hair shaft shortening is observed [42]. Very few studies reported bent hairs in tinea capitis, with an incidence rate between $4 \%$ and $71 \%$ (mean value: 27\%) [6, 19, 20, 42]. They were only observed in patients with ectothrix-type fungal infection $[37,43]$.

Block Hairs and i-Hairs

Block hairs are very short hairs with a transverse horizontal distal end [11]. i-Hairs are block hairs 
Table 1 Trichoscopic features of tinea capitis

\begin{tabular}{llllll}
\hline $\begin{array}{l}\text { Trichoscopic } \\
\text { feature }\end{array}$ & $\begin{array}{l}\text { Reported prevalence in } \\
\text { (mean value }{ }^{\mathbf{b}} \text { ) }\end{array}$ & $\begin{array}{l}\text { Sensitivity }^{\mathrm{c}} \\
(\%)\end{array}$ & $\begin{array}{l}\text { Specificity }^{\mathbf{d}} \\
(\%)\end{array}$ & $\begin{array}{l}\text { Positive predictive } \\
\text { value (\%) }\end{array}$ & $\begin{array}{l}\text { Negative } \\
\text { predictive value } \\
\text { (\%) }\end{array}$ \\
\hline Comma hairs & $13-100(51)$ & 50 & 99 & 94 & 82 \\
$\begin{array}{l}\text { Corkscrew } \\
\text { hairs }\end{array}$ & $14-100(32)$ & 32 & 100 & 98 & 77 \\
$\begin{array}{l}\text { Morse code- } \\
\text { like hairs }\end{array}$ & $12-56(22)$ & 13 & 100 & 100 & 73 \\
Zigzag hairs & $5-49(21)$ & & & & 73 \\
Bent hairs & $4-71(27)$ & 17 & 99 & 83 & 72 \\
Block hairs & $4-50(10)$ & 7 & 100 & 100 & 70 \\
i-Hairs & $4-33(10)$ & 2 & 100 & 83 & 71 \\
\hline
\end{tabular}

a The ranges from original studies $[6,12-24,42]$ are given for trichoscopic features for which the frequency was evaluated

b Mean value was calculated by dividing the total number of patients with a particular feature by the total number of patients in studies reporting the frequency of this feature

c The sensitivity was calculated based on the results from original studies [6, 12-24, 42]

$\mathrm{d}$ The specificity was calculated based on the results from original studies [12-18, 20, 21, 42]

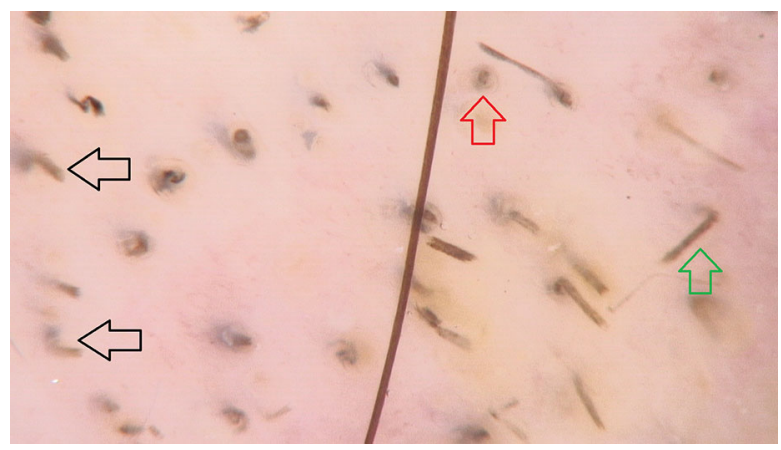

Fig. 2 Trichoscopy of tinea capitis with comma hairs (black arrows), black dots (red arrow) and broken hairs (green arrow) $(\times 70)$

with an accented dark distal end [11]. The terms were introduced by Rudnicka et al. [44]. There were very few studies reporting block hairs and i-hairs in tinea capitis, with an incidence rate of 4-50\% (mean value: 10\%) and 4-33\% (mean value: $10 \%)$ [13, 18-21, 24], respectively. They were also detected in patients with alopecia areata and trichotillomania [45].

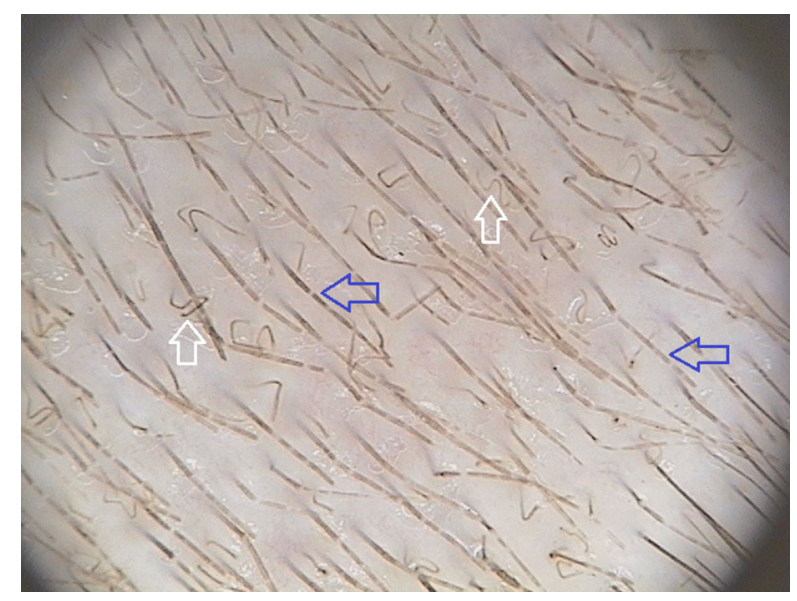

Fig. 3 Trichoscopy of tinea capitis with the presence of Morse code-like hairs (blue arrows) and corkscrew hairs (white arrows) $(\times 20)$

\section{Other Trichoscopic Findings of Tinea Capitis}

Other commonly observed, but not characteristic, trichoscopic findings of tinea capitis include broken hairs, black dots (Fig. 2), and perifollicular and interfollicular scaling. 
Table 2 Trichoscopic differences between Microsporum and Trichophyton tinea capitis

\begin{tabular}{llll}
\hline Trichoscopic feature & $\begin{array}{l}\text { Microsporum tinea capitis } \\
\text { Number of patients }{ }^{\mathbf{a}}(\mathbf{\%})\end{array}$ & $\begin{array}{l}\text { Trichophyton tinea capitis } \\
\text { Number of patients } \mathbf{b}^{\mathbf{b}} \mathbf{( \% )}\end{array}$ & $\begin{array}{l}\text { Statistical significance } \\
(\boldsymbol{p} \text { value })\end{array}$ \\
\hline Comma hairs & $21 / 29(72)$ & $24 / 38(63)$ & 0.42 \\
Corkscrew hairs & $3 / 29(10)$ & $21 / 38(55)$ & $<0.001$ \\
Morse code-like hairs & $8 / 29(28)$ & $0 / 38(0)$ & $<0.001$ \\
Zigzag hairs & $6 / 29(21)$ & $0 / 38(0)$ & $<0.01$ \\
Bent hairs & $4 / 29(14)$ & $0 / 38(0)$ & $<0.05$ \\
Block hairs & $0 / 29(0)$ & $0 / 38(0)$ & - \\
i-Hairs & $0 / 29(0)$ & $0 / 38(0)$ & - \\
Broken hairs & $13 / 29(45)$ & $17 / 38(45)$ & 0.99 \\
Black dots & $3 / 29(10)$ & $3 / 38(8)$ & 0.73 \\
Perifollicular scaling & $3 / 29(10)$ & $2 / 38(5)$ & 0.43 \\
Diffuse scaling & $4 / 29(14)$ & $0 / 38(0)$ & $<0.05$ \\
\hline
\end{tabular}

a 22 cases of Microsporum canis [10, 12, 22, 27, 36, 37, 39, 40, 43, 46-48], 5 cases of Microsporum audouinii [22, 38, 40], 1 case of Microsporum langeronii [23] and 1 case of Microsporum ferrugineum [40]

b 9 cases of Trichophyton violaceum [23, 25, 32, 33, 40, 49], 17 cases of Trichophyton tonsurans [22, 26-30, 43], 4 cases of Trichophyton soudanense [23] and 8 cases of Trichophyton verrucosum [12, 50]

Broken hairs and black dots were observed in both endothrix and ectothrix tinea capitis with an incidence rate of $8-100 \%$ (mean value: $57 \%$ ) $[6,9,12-23,42]$ and $17-80 \%$ (mean value: $34 \%$ ) $[6,12-20,42]$, respectively. In 6-95\% (mean value: $59 \%$ ) $[6,16,19-22]$ and $53-100 \%$ (mean value: $89 \%)[6,14,16,19,20,24,42]$ of patients with tinea capitis perifollicular and diffuse scaling were detected.

\section{Trichoscopic Differences between Microsporum and Trichophyton Tinea Capitis}

In the present analysis Morse code-like hairs, zigzag hairs, bent hairs and diffuse scaling were only present in Microsporum tinea capitis (8/29, $28 \% ; 6 / 29,21 \% ; 4 / 29,14 \%$ and $4 / 29,14 \%$, respectively) ( $p<0.001, p<0.01, p<0.05$ and $p<0.05$, respectively). Conversely, corkscrew hairs were more commonly observed in Trichophyton compared to Microsporum tinea capitis $(21 / 38,55 \%$ vs $3 / 29,10 \%)(p<0.001)$. No significant difference was found in the frequency of comma hairs, black dots, broken hairs, perifollicular scaling, block hairs and i-hairs between Microsporum and Trichophyton tinea capitis.

Detailed data are presented in Table 2.

\section{Trichoscopy in the Monitoring of Treatment Efficacy}

In a study conducted by Campos et al. [19] the disappearance of short broken hairs, corkscrew hairs and zigzag hairs was reported on the 4th week follow-up visit after starting the treatment. At week 8, the decrease of comma hairs, black dots, perifollicular scaling and diffuse scaling was additionally observed. On the 12th week follow-up visit after starting the treatment, dystrophic hairs were not present. However, perifollicular scaling and diffuse scaling were detected. Souissi et al. [36] also reported the disappearance of Morse code-like hairs with the presence of perifollicular scaling and diffuse desquamation on the 4 th week follow-up visit. At this time, the fungal culture was negative. 
Moreover, Richarz et al. [33] presented a case of a patient with disappearance of corkscrew hairs on a follow-up visit after 4 weeks of therapy, with positive direct potassium hydroxide examination and negative fungal culture.

\section{DISCUSSION}

This systematic review aimed to summarize and analyze the usefulness of trichoscopy in tinea capitis. The analysis demonstrated that the presence of characteristic trichoscopic features (comma hairs, corkscrew hairs, Morse code-like hairs, zigzag hairs, bent hairs, block hairs, and $\mathrm{i}$-hairs) is predictive of tinea capitis. According to these observations, trichoscopy may be useful to establish the primary diagnosis of tinea capitis and start the therapy before culture results are available. Moreover, it may be helpful to perform screening in high-risk populations. Broken hairs, black dots, perifollicular and diffuse scaling are commonly observed in tinea capitis. However, they may also be detected in other hair and scalp diseases such as alopecia areata, trichotillomania, lichen planopilaris, discoid lupus erythematosus, seborrheic dermatitis or psoriasis, so they cannot be considered as disease-specific [44].

The present analysis confirmed that trichoscopy is a useful method in differentiating between Microsporum and Trichophyton tinea capitis (Fig. 4), which is important from the perspective of a different therapeutic approach. Terbinafine is considered to be the first-line

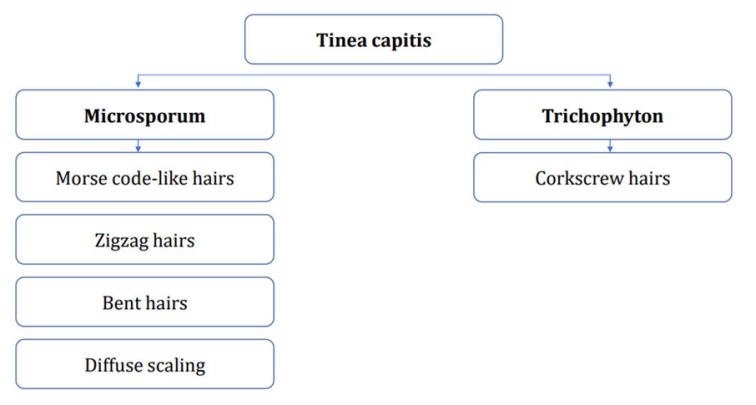

Other trichoscopic findings of both types of tinea capitis include comma hairs, black dots, perifollicular scaling, block hairs and i-hairs

Fig. 4 Characteristic trichoscopic findings of Microsporum and Trichophyton tinea capitis therapy for Trichophyton tinea capitis, while in Microsporum tinea capitis griseofulvin is recommended by many experts [1]. It has been hypothesized that endothrix-type fungal infection (particularly Trichophyton) induces the deformation of the hair shaft without an impairment of the color [31]. Moreover, according to Schechtman et al. [43], in endothrix tinea capitis the hair shaft filled with hyphae tends to break easily near the follicular ostia, so a large number of short comma hairs and black dots may be present in trichoscopic examination. In ectothrix infection (particularly Microsporum) the accumulation of spores around the hair shaft with transverse perforation of the hair shaft is present. This results in the presence of white, thin bands across the hair shaft that form a Morse code-like appearance [31]. Furthermore, transverse fractures of the hair shaft are observed farther from the follicular ostia. Thus, longer corkscrew or zigzag hairs may be observed. In case of a complete fracture, broken hairs may be detected [31]. In the present analysis, corkscrew hairs were more commonly observed in Trichophyton compared with Microsporum tinea capitis. Morse code-like hairs, zigzag hairs, bent hairs and diffuse scaling were only observed in patients with Microsporum tinea capitis. There was no significant difference in the frequency of broken hairs, black dots and comma hairs between Trichophyton and Microsporum tinea capitis.

The role of trichoscopy in monitoring tinea capitis therapy has also been described in the literature $[19,33,36]$. The trichoscopic marker of treatment efficacy is the disappearance of

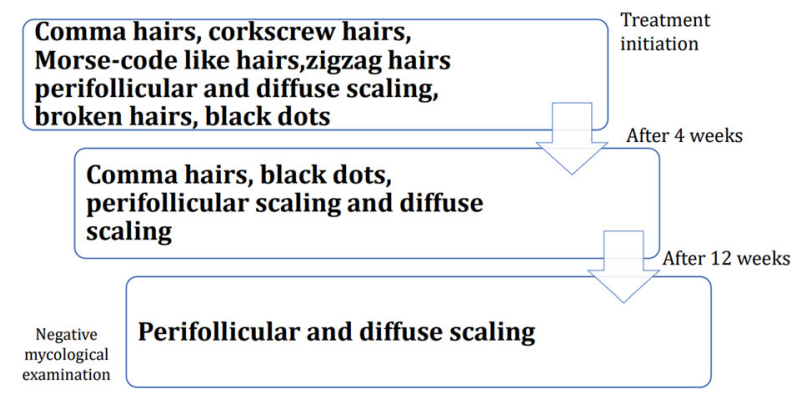

Fig. 5 Trichoscopy of tinea capitis during treatment. Based on Campos et al. [19], Richarz et al. [33] and Souissi et al. [36] 
dystrophic hairs (comma hairs, corkscrew hairs, zigzag hairs, Morse code-like hairs, broken hairs and black dots), 4-12 weeks after therapy initiation $[19,33,36]$. Perifollicular and diffuse scaling tends to resolve more slowly compared to hair shaft abnormalities (Fig. 5). Therefore, it cannot be considered to be a marker of therapy failure. Moreover, previous data suggested that trichoscopy may be more reliable than repeated potassium hydroxide examinations in the monitoring of treatment efficacy in tinea capitis [33].

A limitation of our review was due to the small size of the group of patients in the analysis of trichoscopic differences between Microsporum and Trichophyton tinea capitis.

\section{CONCLUSIONS}

Trichoscopy is a useful method in the diagnosis of tinea capitis. Comma hairs, corkscrew hairs, Morse code-like hairs, zigzag hairs, bent hairs, block hairs and i-hairs are diagnostic trichoscopic markers of tinea capitis. Trichoscopy may be a helpful method in the differentiation between Microsporum and Trichophyton tinea capitis, and consequently, the selection of the appropriate therapy. Finally, it can be applied to the monitoring of treatment efficacy.

\section{ACKNOWLEDGEMENTS}

Funding. No funding or sponsorship was received for this study or publication of this article.

Authorship. All named authors meet the International Committee of Medical Journal Editors (ICMJE) criteria for authorship for this article, take responsibility for the integrity of the work as a whole, and have given their approval for this version to be published.

Disclosures. Anna Waśkiel-Burnat, Adriana Rakowska, Mariusz Sikora, Piotr Ciechanowicz, Małgorzata Olszewska and Lidia Rudnicka have nothing to disclose. Adriana Rakowska is a member of the journals Editorial Board.

Compliance with Ethics Guidelines. This article is based on previously conducted studies and does not contain any studies with human participants or animals performed by any of the authors.

Data Availability. The datasets during and/ or analyzed during the current study are available from the corresponding author on reasonable request.

Open Access. This article is licensed under a Creative Commons Attribution-NonCommercial 4.0 International License, which permits any non-commercial use, sharing, adaptation, distribution and reproduction in any medium or format, as long as you give appropriate credit to the original author(s) and the source, provide a link to the Creative Commons licence, and indicate if changes were made. The images or other third party material in this article are included in the article's Creative Commons licence, unless indicated otherwise in a credit line to the material. If material is not included in the article's Creative Commons licence and your intended use is not permitted by statutory regulation or exceeds the permitted use, you will need to obtain permission directly from the copyright holder. To view a copy of this licence, visit http://creativecommons.org/licenses/by$\mathrm{nc} / 4.0 /$.

\section{REFERENCES}

1. Fulle LC, Barton RC, Mohd Mustapa MF, et al. British Association of Dermatologists' guidelines for the management of tinea capitis. 2014. Br J Dermatol. 2014;171:454-63.

2. Ginter-Hanselmayer G, Weger W, Ilkit M, Smolle J. Epidemiology of tinea capitis in Europe: current state and changing patterns. Mycoses. 2007; 50(Suppl 2):6-13.

3. Gupta AK, Mays RR, Versteeg SG, et al. Tinea capitis in children: a systematic review of management. J Eur Acad Dermatol Venereol. 2018;32:2264-74. 
4. Adesiji YO, Omolade FB, Aderibigbe IA et al. Prevalence of tinea capitis among children in Osogbo, Nigeria, and the associated risk factors. Diseases 2019;7.

5. Michaels BD, Del Rosso JQ. Tinea capitis in infants: recognition, evaluation, and management suggestions. J Clin Aesthet Dermatol. 2012;5:49-59.

6. Aqil N, BayBay H, Moustaide K, et al. A prospective study of tinea capitis in children: making the diagnosis easier with a dermoscope. J Med Case Rep. 2018;12:383.

7. Lacarrubba F, Micali G, Tosti A. Scalp dermoscopy or trichoscopy. Curr Probl Dermatol. 2015;47: 21-32.

8. Rudnicka L, Olszewska M, Rakowska A, KowalskaOledzka E, Slowinska M. Trichoscopy: a new method for diagnosing hair loss. J Drugs Dermatol. 2008;7:651-4.

9. Dhaille F, Dillies AS, Dessirier F et al. A single typical trichoscopic feature is predictive of tinea capitis-a prospective multicenter study. Br J Dermatol 2019.

10. Slowinska M, Rudnicka L, Schwartz RA, et al. Comma hairs: a dermatoscopic marker for tinea capitis: a rapid diagnostic method. J Am Acad Dermatol. 2008;59:S77-9.

11. Rudnicka L, Rakowska A, Kerzeja M, Olszewska M. Hair shafts in trichoscopy: clues for diagnosis of hair and scalp diseases. Dermatol Clin. 2013;31(695-708):x.

12. Ekiz O, Sen BB, Rifaioglu EN, Balta I. Trichoscopy in paediatric patients with tinea capitis: a useful method to differentiate from alopecia areata. J Eur Acad Dermatol Venereol. 2014;28:1255-8.

13. Rakowska A, Slowinska M, Olszewska M, Rudnicka L. New trichoscopy findings in trichotillomania: flame hairs, V-sign, hook hairs, hair powder, tulip hairs. Acta Derm Venereol. 2014;94:303-6.

14. Nikam VV, Mehta HH. A nonrandomized study of trichoscopy patterns using nonpolarized (contact) and polarized (noncontact) dermatoscopy in hair and shaft disorders. Int J Trichol. 2014;6:54-62.

15. El-Taweel AE, El-Esawy F, Abdel-Salam O. Different trichoscopic features of tinea capitis and alopecia areata in pediatric patients. Dermatol Res Pract. 2014;2014:848763.

16. Park J, Kim JI, Kim HU, Yun SK, Kim SJ. Trichoscopic findings of hair loss in Koreans. Ann Dermatol. 2015;27:539-50.
17. Chiramel MJ, Sharma VK, Khandpur S, Sreenivas V. Relevance of trichoscopy in the differential diagnosis of alopecia: a cross-sectional study from North India. Indian J Dermatol Venereol Leprol. 2016;82: 651-8.

18. Amer M, Helmy A, Amer A. Trichoscopy as a useful method to differentiate tinea capitis from alopecia areata in children at Zagazig University Hospitals. Int J Dermatol. 2017;56:116-20.

19. Campos S, Brasileiro A, Galhardas C, et al. Followup of tinea capitis with trichoscopy: a prospective clinical study. J Eur Acad Dermatol Venereol. 2017;31:e478-80.

20. Brasileiro A, Campos S, Cabete J, et al. Trichoscopy as an additional tool for the differential diagnosis of tinea capitis: a prospective clinical study. Br J Dermatol. 2016;175:208-9.

21. Al-Refu K. Clinical significance of trichoscopy in common causes of hair loss in children: analysis of 134 cases. Int J Trichol. 2018;10:154-61.

22. Isa RAB, Pimentel MI, Arenas R, Tosti A, Cruz AC. Dermoscopy in tinea capitis: a prospective study on 43 patients. Medicina Cutanea Ibero-Latino-Americana. 2014;42:18-22.

23. Hughes R, Chiaverini C, Bahadoran P, Lacour JP. Corkscrew hair: a new dermoscopic sign for diagnosis of tinea capitis in black children. Arch Dermatol. 2011;147:355-6.

24. Moneib HA, El-Shiemy SMH, Saudi WM, et al. Hair loss among a group of Egyptian children: a clinical and dermoscopic study. J Egypt Women's Dermatol Soc. $2017 ; 14: 9-24$.

25. Lu M, Ran Y, Dai Y, et al. An ultrastructural study on corkscrew hairs and cigarette-ash-shaped hairs observed by dermoscopy of tinea capitis. Scanning. 2016;38:128-32.

26. Neri I, Starace M, Patrizi A, Balestri R. Corkscrew hair: a trichoscopy marker of tinea capitis in an adult white patient. JAMA Dermatol. 2013;149: 990-1.

27. Lekkas D, Ioannides D, Apalla Z, et al. Dermoscopy for discriminating between Trichophyton and Microsporum infections in tinea capitis. J Eur Acad Dermatol Venereol. 2018;32:e234-5.

28. Nguyen J CL, Smith J. Trichoscopic features of tinea capitis in a straight-haired hispanic woman. Dermatol Online J. 2018;24.

29. Pinheiro AM, Lobato LA, Varella TC. Dermoscopy findings in tinea capitis: case report and literature review. An Bras Dermatol. 2012;87:313-4. 
30. Razmi TM, De D, Vinay K. 'Corkscrews' on the patchy alopecia of a girl. Arch Dis Child Educ Pract Ed 2018.

31. Bourezane $Y$, Bourezane Y. Analysis of trichoscopic signs observed in 24 patients presenting tinea capitis: hypotheses based on physiopathology and proposed new classification. Ann Dermatol Venereol. 2017;144:490-6.

32. Vazquez-Lopez F, Palacios-Garcia L, Argenziano G. Dermoscopic corkscrew hairs dissolve after successful therapy of Trichophyton violaceum tinea capitis: a case report. Australas J Dermatol. 2012;53: 118-9.

33. Richarz NA, Barboza L, Monsonis M, GonzalezEnsenat MA, Vicente A. Trichoscopy helps to predict the time point of clinical cure of tinea capitis. Australas J Dermatol. 2018;59:e298-9.

34. Rudnicka L, Olszewska M, Rakowska A, Slowinska M. Trichoscopy update 2011. J Dermatol Case Rep. 2011;5:82-8.

35. Rudnicka L, Olszewska M, Waskiel A, Rakowska A. Trichoscopy in hair shaft disorders. Dermatol Clin. 2018;36:421-30.

36. Souissi A, Ben Lagha I, Toukabri N, Mama M, Mokni M. Morse code-like hairs in tinea capitis disappear after successful treatment. Int J Dermatol. 2018;57: e150-1.

37. Lacarrubba F, Verzi AE, Micali G. Newly described features resulting from high-magnification dermoscopy of tinea capitis. JAMA Dermatol. 2015;151:308-10.

38. Wang HH, Lin YT. Bar code-like hair: dermoscopic marker of tinea capitis and tinea of the eyebrow. J Am Acad Dermatol. 2015;72:S41-2.

39. Tang J, Ran X, Ran Y. Ultraviolet dermoscopy for the diagnosis of tinea capitis. J Am Acad Dermatol. 2017;76:S28-30.

40. Lin Y-T, Li Y-C. The dermoscopic comma, zigzag, and bar code-like hairs: Markers of fungal infection of the hair follicles. 2013.
41. Khunkhet S, Vachiramon V, Suchonwanit P. Trichoscopic clues for diagnosis of alopecia areata and trichotillomania in Asians. Int J Dermatol. 2017;56: 161-5.

42. Shim WH, Jwa SW, Song M, et al. Dermoscopic approach to a small round to oval hairless patch on the scalp. Ann Dermatol. 2014;26:214-20.

43. Schechtman RC, Silva ND, Quaresma MV, et al. Dermatoscopic findings as a complementary tool in the differential diagnosis of the etiological agent of tinea capitis. An Bras Dermatol. 2015;90:13-5.

44. Rudnicka L, Olszewska M, Rakowska A. Atlas of trichoscopy: dermoscopy in hair and scalp disease. London: Springer; 2012.

45. Malakar S, Mehta PR. "i hair": a prognostic marker in alopecia areata \& trichotillomania. Indian J Dermatol. 2017;62:658-60.

46. Errichetti E, Stinco G. Dermoscopy as a useful supportive tool for the diagnosis of pityriasis amiantacea-like tinea capitis. Dermatol Pract Concept. 2016;6:63-5.

47. Veasey JV, Muzy GSC. Tinea capitis: correlation of clinical presentations to agents identified in mycological culture. An Bras Dermatol. 2018;93: 465-6.

48. Vastarella M, Gallo L, Cantelli M, Nappa P, Fabbrocini G. An undetected case of tinea capitis in an elderly woman affected by dermatomyositis: how trichoscopy can guide to the right diagnosis. Skin Appendage Disorders 2019.

49. Mapelli ET, Gualandri L, Cerri A, Menni S. Comma hairs in tinea capitis: a useful dermatoscopic sign for diagnosis of tinea capitis. Pediatr Dermatol. 2012;29:223-4.

50. Toledo-Pastrana T, Hernandez MJ, Camacho Martinez FM. Perifollicular erythema as a trichoscopy sign of progression in frontal fibrosing alopecia. Int J Trichology. 2013;5:151-3. 